Neuropsychology, Neuropsychiatry, and Behavioral Neurology 


\section{Critical Issues in Neuropsychology}

Series Editors

Antonio E. Puente

University of North Carolina, Wilmington
Cecil R. Reynolds

Texas A\&M University

\section{ASSESSMENT ISSUES IN CHILD NEUROPSYCHOLOGY}

Edited by Michael G. Tramontana and Stephen R. Hooper

BRAIN MECHANISMS IN PROBLEM SOLVING AND

INTELLIGENCE: A Lesion Survey of the Rat Brain

Robert Thompson, Francis M. Crinella, and Jen Yu

BRAIN ORGANIZATION OF LANGUAGE AND COGNITIVE PROCESSES

Edited by Alfredo Ardila and Feggy Ostrosky-Solis

HANDBOOK OF CLINICAL CHILD NEUROPSYCHOLOGY

Edited by Cecil R. Reynolds and Elaine Fletcher-Janzen

MEDICAL NEUROPSYCHOLOGY: The Impact of Disease on Behavior Edited by Ralph E. Tarter, David H. Van Thiel, and Kathleen L. Edwards

NEUROPSYCHOLOGICAL FUNCTION AND BRAIN IMAGING

Edited by Erin D. Bigler, Ronald A. Yeo, and Eric Turkheimer

NEUROPSYCHOLOGY, NEUROPSYCHIATRY, AND BEHAVIORAL NEUROLOGY

Rhawn Joseph

RELIABILITY AND VALIDITY IN NEUROPSYCHOLOGICAL ASSESSMENT

Michael D. Franzen

A Continuation Order Plan is available for this series. A continuation order will bring delivery of each new volume immediately upon publication. Volumes are billed only upon actual shipment. For further information please contact the publisher. 


\section{Neuropsychology, \\ Neuropsychiatry, and Behavioral Neurology}

\section{Rhawn Joseph}

Neurobehavioral Center

Santa Clara, California 


\section{Library of Congress Cataloging in Publication Data}

Joseph, Rhawn.

Neuropsychology, neuropsychiatry, and behavioral neurology / Rhawn Joseph.

p. cm. - (Critical issues in neuropsychology)

Includes bibliographical references.

ISBN 978-1-4419-3211-2 ISBN 978-1-4757-5969-3 (eBook)

DOI 10.1007/978-1-4757-5969-3

1. Neuropsychiatry. 2. Neuropsychology. I. Title. II. Series. [DNLM: 1. Neurophysiology. 2. Neuropsychology. 3. Psychiatry. WL 103 J83n]

RC341.J67 1989

616.8-dc20

DNLM/DLC

89-16319

for Library of Congress

CIP

$\begin{array}{lllllll}10 & 9 & 8 & 7 & 6 & 5 & 4\end{array}$

(C) 1990 Springer Science+Business Media New York

Originally published by Plenum Press, New York in 1990

All rights reserved

No part of this book may be reproduced, stored in a retrieval system, or transmitted in any form or by any means, electronic, mechanical, photocopying, microfilming, recording, or otherwise, without written permission from the Publisher 
To those who say

"NO!"

I say

"YES!" 


\section{Preface}

This book is written for the clinician, students, and practitioners of neuropsychology, neuropsychiatry, and behavioral neurology. It has been my intent throughout to present a synthesis of ideas and research findings. I have reviewed thousands of articles and research reports and have drawn extensively from diverse sources in philosophy, psychology, neurology, neurosurgery, neuropsychiatry, physiology, and neuroanatomy in order to produce this text. Of course I have also drawn from my own experience as a clinician and research scientist in preparing this work and in this regard some of my own biases and interests are represented.

I have long sought to understand the human mind and the phenomena we experience as conscious awareness. After many years of studying a variety of Western and Eastern psychologists and philosophers, including the Buddhist, Taoist, and Hindu philosophical systems, I began, while still an undergraduate student, to formulate my own theory of the mind. I felt, though, that what I had come upon were only pieces of half the puzzle. What I knew of the brain was minimal. Indeed, it came as quite a surprise when one day I came across the journal Brain as I was browsing through the periodicals section of the library. I was awed. An entire journal devoted to the brain was quite a revelation. Nevertheless, although intrigued by the possibilities, I resisted. What did the brain have to do with "consciousness," emotion, or even clinical psychology? I was to find out soon thereafter, when I met and became good friends with Dr. David Duval, then a graduate student. He was both biologist and philosopher and told me about a new experimental memory and brain injury rehabilitation clinic at the Palo Alto Veterans Administration Hospital, where he had just begun to work. I was intrigued by his stories and one day accompanied him as an invited visitor. (Later I obtained a position at the clinic for a short time.) I was astounded and deeply impressed by the seemingly bizarre variety of symptoms demonstrated by many of the brain-injured vets I met that day, and I was shocked to be confronted with what appeared to be an undeniable truth: "If you damage the brain you damage consciousness." On that day I began a quest to understand the brain and the manner in which it interacts so as to produce consciousness, awareness, personality, memory, thinking, emotion, neurosis, and psychosis.

That was almost 17 years ago. Since that time, I have been very fortunate to have had a number of opportunities to test and observe over a thousand brain-injured individuals and to conduct a variety of studies in the neurosciences. These have included animal research in neurophysiology, developmental neuroanatomy, and sensory deprivation; 
human neuropsychological studies conducted on children and split-brain, brain-injured, and neurosurgical patients; and research on hormonal and early environmental influences on arousal, aggression, memory, and sex differences in cognition.

In conducting these studies my knowledge and understanding of brain psychiatry and neuropsychology have greatly expanded. Nevertheless, many of the books in the neurosciences treat psychiatry, neurology, and neuropsychology as if they were separate and mutually exclusive fields. It is my belief that these areas of study are in fact one and the same. I think it is important to realize that what appears to be a "manic" disorder may in fact be a manifestation of right frontal lobe disease, or that the patient who suddenly develops "schizophrenia" may instead have suffered a stroke involving the basal ganglia or left temporal lobe. I believe it is also important to recognize not only that diverse symptoms may be indicative of localized dysfunction but also how specific abnormalities can be secondary to disconnection syndromes, disinhibition, epileptic disturbances, and/or tumors, strokes, head injury, and diffuse physiological changes. To understand and recognize these problems it is necessary to have at least some knowledge of neuroanatomy and the manner in which various nuclei and brain regions interact. These are just some of the issues detailed in the pages that follow.

Santa Clara, California

Rhawn Joseph 


\section{Acknowledgments}

This book is written for the clinician. However, it draws from a number of research and theoretical perspectives as well as my own experiences in diverse neuroscience settings, including my readings in the neurosciences, existential phenomenology, and psychoanalysis. Nietzsche, Freud, Jung, Sartre, Geschwind, Luria, and many others have greatly contributed to my thought and understanding of the brain, cognition, emotion, and conscious awareness. Drs. Oakley Ray, Peter Koestenbaum, Arlene Kasprisin, Arthur Anderson, and Roberta Gallagher have contributed significantly to my philosophical and experiental growth. Drs. Oakley Ray, Vivian Casagrande, J. Kass, and J. Siegel provided me with tremendous opportunities to undertake a broad range of neuroscience research, whereas Drs. A. Kasprisin, J. Dalton, J. Gonen, R. Delaney, R. Novelly, M. Andersen, and $\mathrm{A}$. Anton provided me with opportunities to examine a wide variety of brain-injured, neurosurgical, split-brain, and psychiatric patients. Others who have contributed significantly include Drs. Edith Kaplin, S. Berenbaum, D. O'Leary, Ralph Peterson, and Joe Ryan; the Psychiatry and Neurology Departments at UHS/The Chicago Medical School and the North Chicago Veterans Administration Medical Center; the Neurology and Neurosurgery staff at West Haven Veterans Administration Medical Center and Yale University Medical School; the staff at the Veterans Administration/Yale Seizure Unit; the Journal of Clinical Psychology for allowing me to republish portions of my 1982 monograph and a slightly expanded version of my 1988 monograph; and Fran Bernstein and the San Jose State University Library staff.

In preparing for this book I also spent more than a few hours browsing through old editions of various periodicals. I was often fascinated by what I discovered hidden away and uncited. I once read that "the only new ideas are those which have been forgotten." I would amend that to read "many new ideas...." This is certainly apparent after reading authors such as Hughlings Jackson and many of his contemporaries, who lived and wrote during the late $1800 \mathrm{~s}$, as well as other researchers and scholars publishing as recently as the 1940s. Although the field of neuropsychology has grown phenomenally during the last 25 years, a good deal of the groundwork was brilliantly proposed and explored long ago. Nevertheless much remains to be explored and discovered. It is my hope that this neurosynthesis of old and new will contribute to the making of many new discoveries. I very much appreciate the assistance and patience of my two editors Steve Melvin and Eliot Werner. 


\section{Contents}

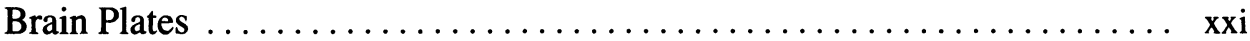

Chapter 1

The Right Cerebral Hemisphere: Emotion, Music, Visual-Spatial Skills, Body Image, Dreams, and Awareness ................... 1

Left Hemisphere Overview ............................... 1

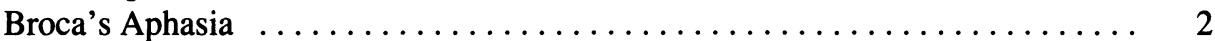

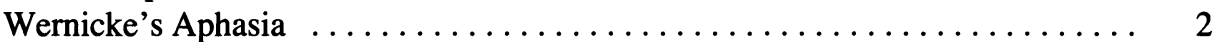

Right Cerebral Hemisphere $\ldots \ldots \ldots \ldots \ldots \ldots \ldots \ldots \ldots \ldots \ldots \ldots \ldots, \quad 4$

Comprehension and Expression of Emotional Speech $\ldots \ldots \ldots \ldots \ldots \ldots \ldots \quad 4$

Right Hemisphere Emotional Language Axis $\ldots \ldots \ldots \ldots \ldots \ldots \ldots \ldots, 6$

Music and Nonverbal Environmental Sounds $\ldots \ldots \ldots \ldots \ldots \ldots \ldots \ldots, 8$

Constructional and Spatial-Perceptual Skills $\ldots \ldots \ldots \ldots \ldots \ldots \ldots \ldots, 12$

Inattention and Visual-Spatial Neglect $\ldots \ldots \ldots \ldots \ldots \ldots \ldots \ldots \ldots \ldots, 16$

Disturbances of the Body Image $\ldots \ldots \ldots \ldots \ldots \ldots \ldots \ldots \ldots \ldots \ldots, 18$

Facial-Emotional Recognition and Prosopagnosia $\ldots \ldots \ldots \ldots \ldots \ldots \ldots, 22$

Disturbances of Emotion and Personality $\ldots \ldots \ldots \ldots \ldots \ldots \ldots \ldots \ldots, 23$

Consciousness, Awareness, Memory, and Dreaming $\ldots \ldots \ldots \ldots \ldots \ldots \ldots 25$

Right Hemisphere Mental Functioning $\ldots \ldots \ldots \ldots \ldots \ldots \ldots \ldots \ldots \ldots .25$

Right Brain Perversity .................................. 27

Lateralized Goals and Attitudes $\ldots \ldots \ldots \ldots \ldots \ldots \ldots \ldots \ldots \ldots \ldots .29$

Lateralized Memory Functioning $\ldots \ldots \ldots \ldots \ldots \ldots \ldots \ldots \ldots \ldots \ldots .29$

Dreaming and Hemispheric Oscillation $\ldots \ldots \ldots \ldots \ldots \ldots \ldots \ldots \ldots, 31$

Long-Lost Childhood Memories $\ldots \ldots \ldots \ldots \ldots \ldots \ldots \ldots \ldots \ldots \ldots, 34$

Emotional and Right Brain Functioning in Children ............... 34

Functional Commissurotomies and Limited Interhemispheric Transfer ...... 36

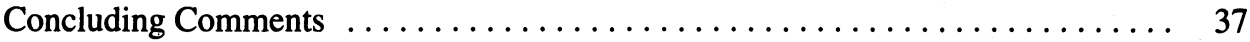

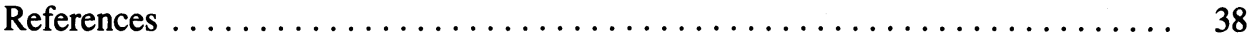




\section{Chapter 2}

The Left Cerebral Hemisphere: Aphasia, Alexia, Agraphia, Agnosia, Apraxia, Language, and Thought $\ldots \ldots \ldots \ldots \ldots \ldots \ldots \ldots \ldots \ldots$

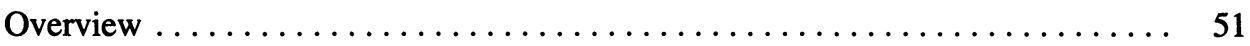

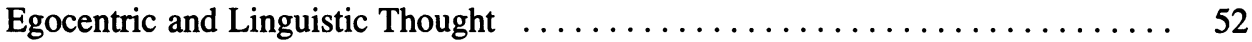

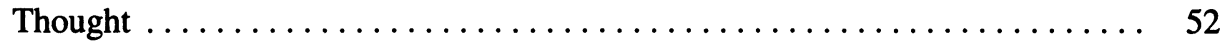

The Development of Language and Thought $\ldots \ldots \ldots \ldots \ldots \ldots \ldots \ldots \ldots$

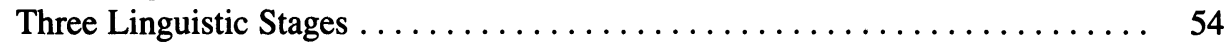

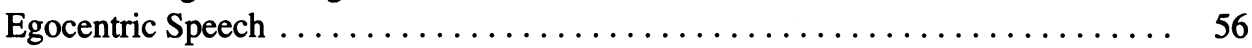

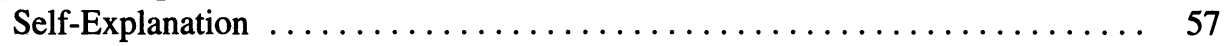

Interhemispheric Communication $\ldots \ldots \ldots \ldots \ldots \ldots \ldots \ldots \ldots \ldots \ldots \ldots$

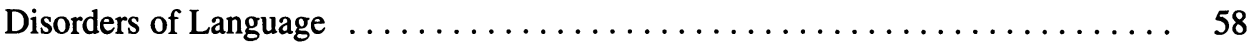

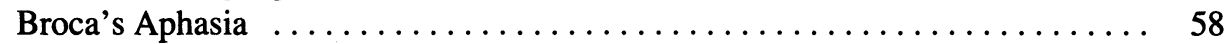

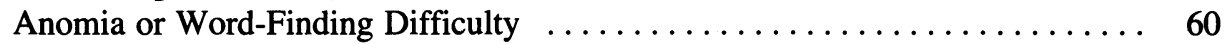

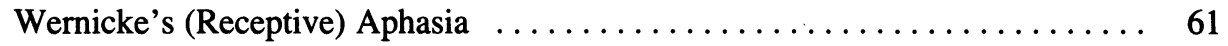

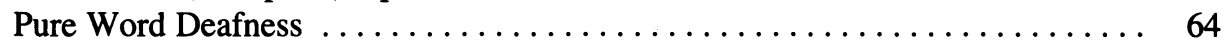

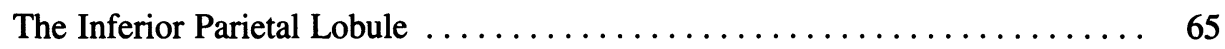

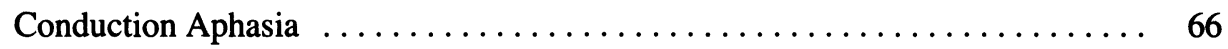

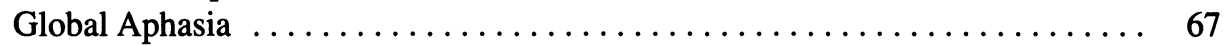

Isolation of the Speech Area (Transcortical Aphasia) . . . . . . . . . . 67

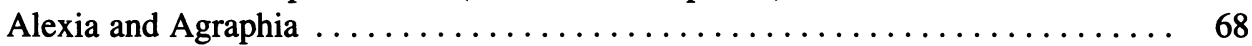

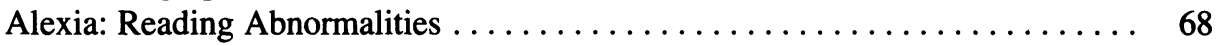

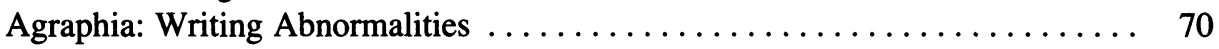

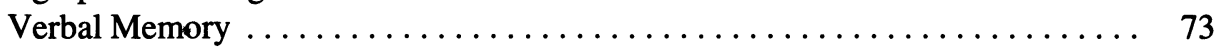

Language and Temporal-Sequential Motor Control $\ldots \ldots \ldots \ldots \ldots \ldots \ldots \ldots 74$

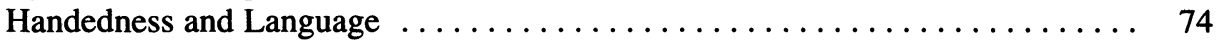

Naming, Knowing, Counting, Finger Recognition, and Hand Control . . . . . 75

Agnosia, Apraxia, Acalculia, and Orientation in Space .............. 76

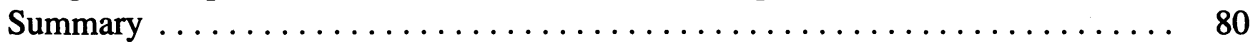

References ..................................... 80

Chapter 3

The Limbic System: Emotion, Laterality, and Unconscious Mind ..... 87

Affective Origins: Olfaction and Somesthesis $\ldots \ldots \ldots \ldots \ldots \ldots \ldots \ldots \ldots \ldots$

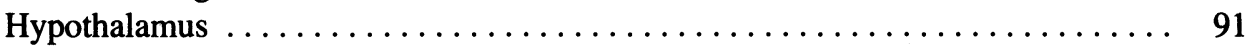

Sexual Dimorphism in the Hypothalamus $\ldots \ldots \ldots \ldots \ldots \ldots \ldots \ldots \ldots . \ldots 1$

Lateral and Ventromedial Hypothalamic Nuclei ................ 92

Hypothalamic Damage and Emotional Incontinence: Laughter and Rage . . . . 95

Psychic Manifestations of Hypothalamic Activity ................ 97

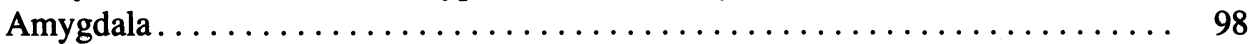

Medial and Lateral Amygdaloid Nuclei $\ldots \ldots \ldots \ldots \ldots \ldots \ldots \ldots \ldots \ldots$

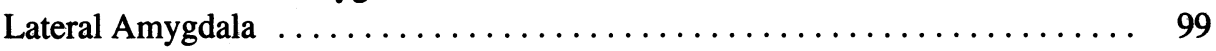

Fear, Rage, and Aggression $\ldots \ldots \ldots \ldots \ldots \ldots \ldots \ldots \ldots \ldots \ldots \ldots \ldots \ldots$ 
Social-Emotional Agnosia $\ldots \ldots \ldots \ldots \ldots \ldots \ldots \ldots \ldots \ldots \ldots \ldots \ldots$

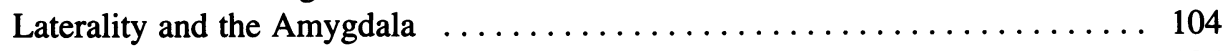

Emotion and Temporal Lobe Seizures $\ldots \ldots \ldots \ldots \ldots \ldots \ldots \ldots \ldots \ldots \ldots$

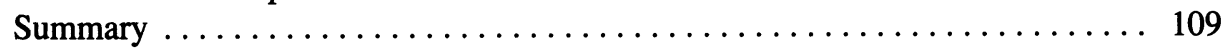

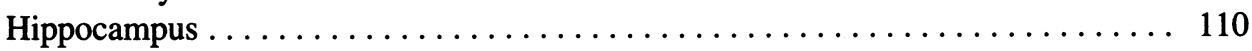

Hippocampal Arousal, Attention, and Inhibitory Influences $\ldots \ldots \ldots \ldots \ldots 112$

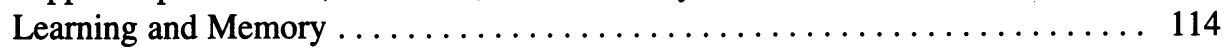

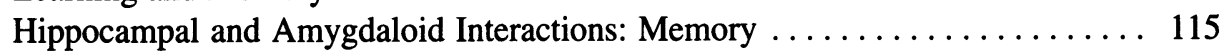

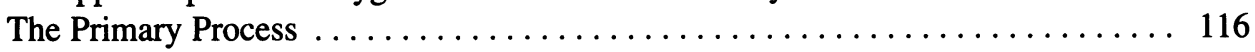

Amygdaloid-Hippocampal Interactions during Infancy $\ldots \ldots \ldots \ldots \ldots \ldots 116$

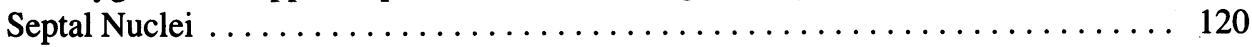

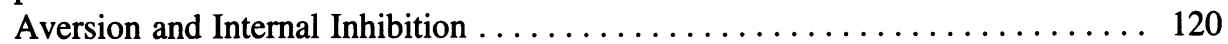

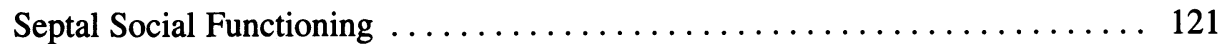

Attachment and Amygdaloid-Septal Developmental Interactions . . . . . . 122

Deprivation and Amygdaloid-Septal Functioning ............... 124

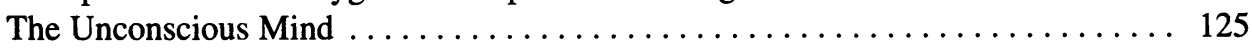

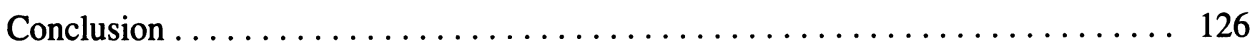

References .................................. 127

\section{Chapter 4}

The Frontal Lobes: Neuropsychiatry, Neuropsychology, and Behavioral

Neurology ............................... 139

Motor Regions of the Frontal Lobes $\ldots \ldots \ldots \ldots \ldots \ldots \ldots \ldots \ldots \ldots \ldots \ldots$

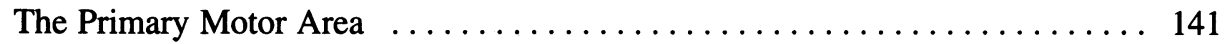

The Premotor Cortex ............................. 142

The Supplementary Motor Area and the Medial Frontal Lobes .......... 143

The Frontal-Medial Walls of the Cerebral Hemispheres . . . . . . . . . . . 144

The Anterior Cingulate . . . . . . . . . . . . . . . . . . . . 149

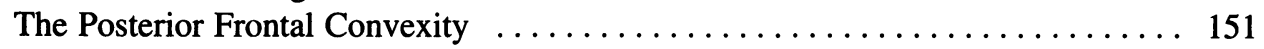

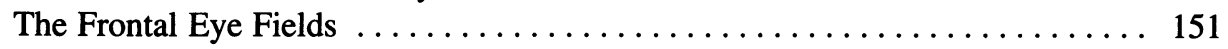

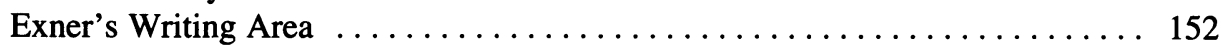

Broca's Speech Area .................................... 153

Confabulation and Right Frontal Emotional and Prosodic Speech ......... 155

The Orbital Frontal Lobes and Inferior Convexity . . . . . . . . . . . . . . . . 157

The Orbital Frontal Lobes . . . . . . . . . . . . . . . . . . . . . . . . . . . 157

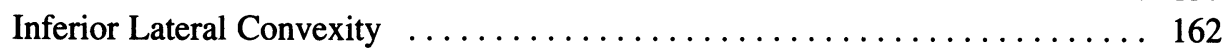

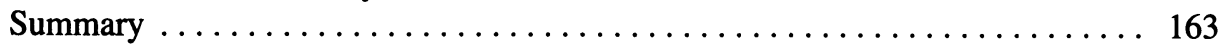

Lateral-Frontal Cortical Monitoring $\ldots \ldots \ldots \ldots \ldots \ldots \ldots \ldots \ldots \ldots \ldots$

Frontal-Thalamic Control of Cortical Activity . . . . . . . . . . . . . . . 164

Personality and Behavioral Alterations Secondary to Frontal Injury . . . . . . . 167

The Frontal Lobe Personality . . . . . . . . . . . . . . . . . . . . . . . . . . . . . 167

Apathy and Pseudodepressive Features $\ldots \ldots \ldots \ldots \ldots \ldots \ldots \ldots \ldots \ldots \ldots$

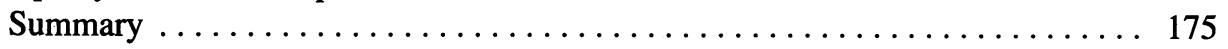


The Right and Left Frontal Lobes $\ldots \ldots \ldots \ldots \ldots \ldots \ldots \ldots \ldots \ldots \ldots \ldots \ldots \ldots$

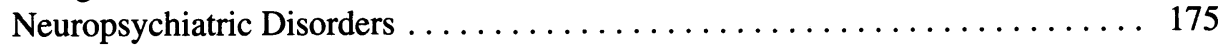

Right Frontal Dominance for Arousal $\ldots \ldots \ldots \ldots \ldots \ldots \ldots \ldots \ldots \ldots \ldots$

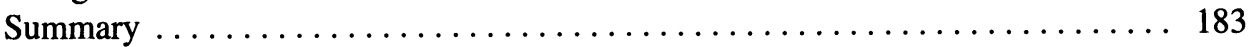

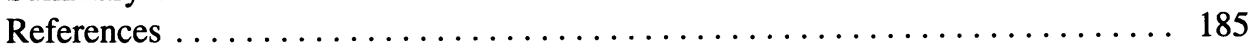

\section{Chapter 5}

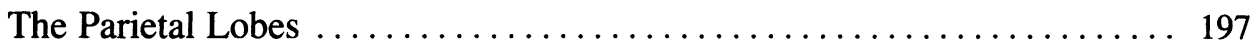

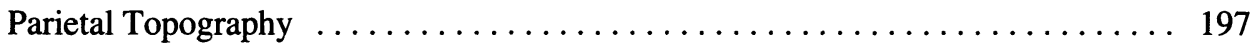

The Primary Somesthetic Receiving Areas $\ldots \ldots \ldots \ldots \ldots \ldots \ldots \ldots \ldots \ldots . \ldots \ldots$

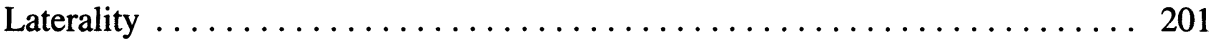

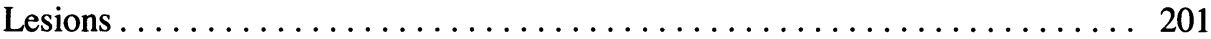

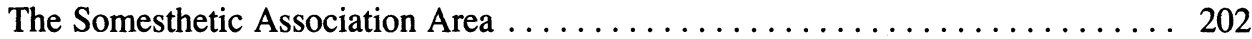

Hand-Manipulation Cells ........................... 202

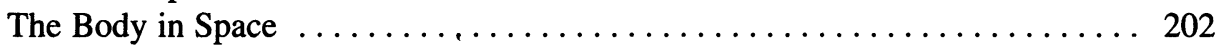

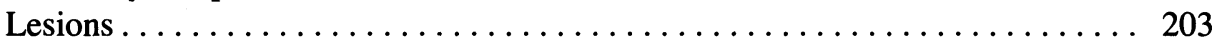

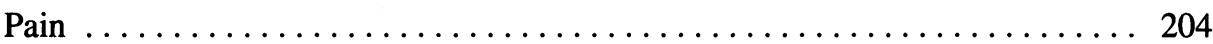

Area 7 and the Superior-Posterior Parietal Lobule ................... 204

Polymodal Information Processing ........................... 204

Three-Dimensional Analysis of Body-Spatial Interaction . . . . . . . . . 205

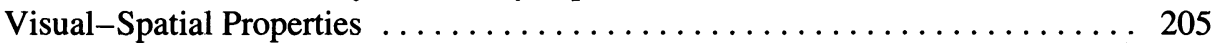

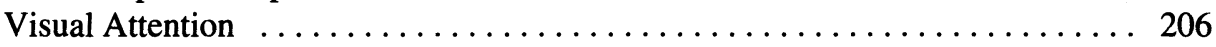

Motivational and Grasping Functions $\ldots \ldots \ldots \ldots \ldots \ldots \ldots \ldots \ldots \ldots \ldots \ldots \ldots$

Lesions and Laterality . . . . . . . . . . . . . . . . . . . . . 207

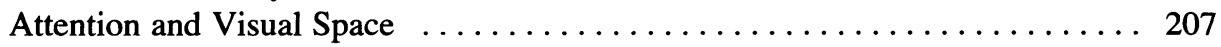

Localization of Objects in Space ........................ 207

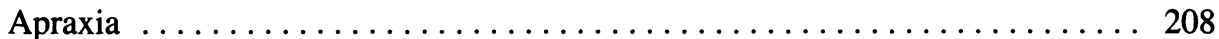

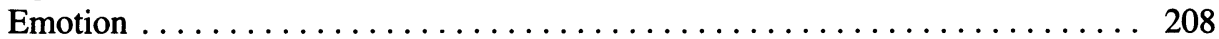

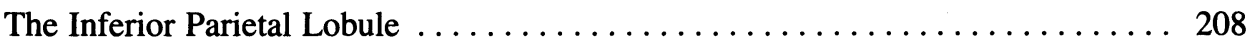

The Multimodal Assimilation Area . . . . . . . . . . . . . . . . . . . . . . . 209

Language Capabilities .............................. 209

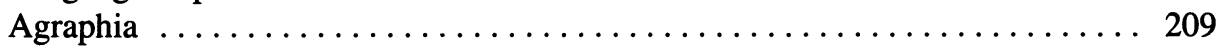

Lateralized Temporal-Sequential Functions $\ldots \ldots \ldots \ldots \ldots \ldots \ldots \ldots \ldots$

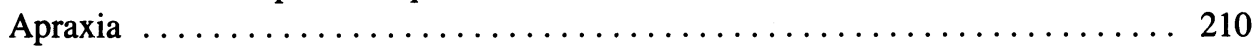

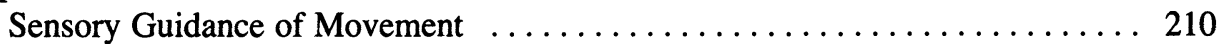

Apraxic Abnormalities ............................. 211

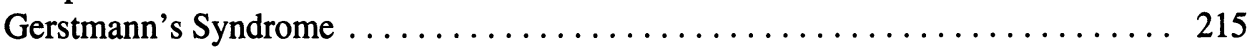

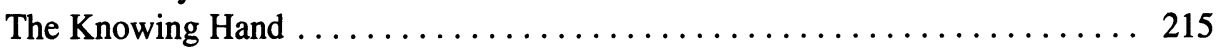

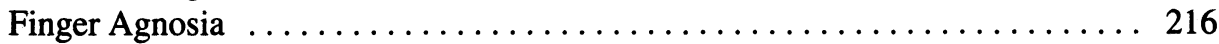

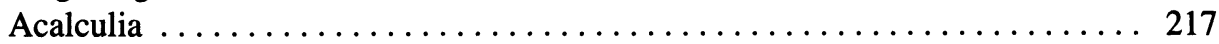

Pure Acalculia/Anarithmetria ......................... 217

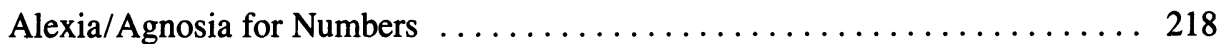

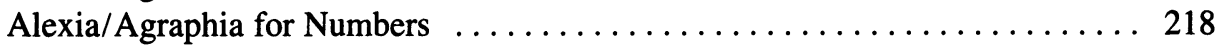

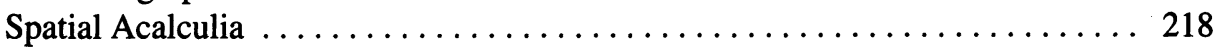

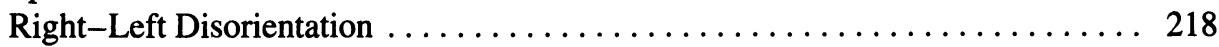


CONTENTS XV XV X

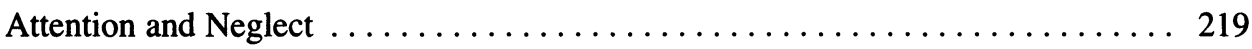

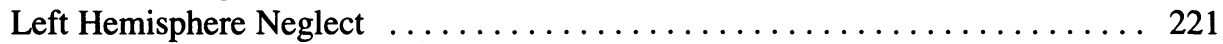

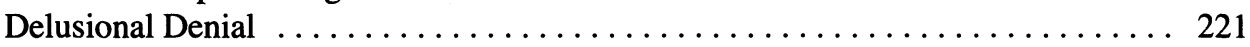

Disconnection, Confabulation, and Gap Filling ............... 222

Delusional Playmates and Egocentric Speech ................... 223

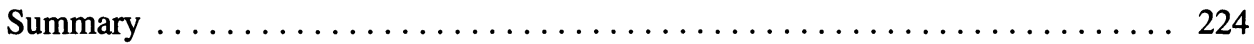

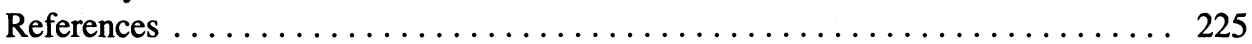

\section{Chapter 6}

The Occipital Lobe $\ldots \ldots \ldots \ldots \ldots \ldots \ldots \ldots \ldots \ldots \ldots \ldots \ldots \ldots$

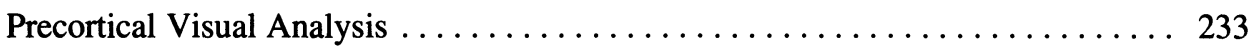

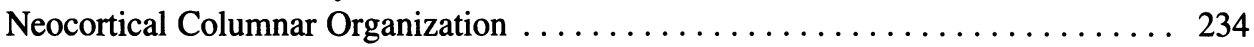

Simple, Complex, and Hypercomplex Cells .................. 234

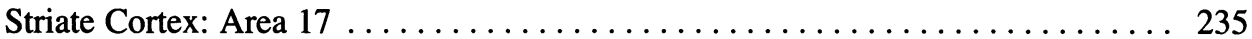

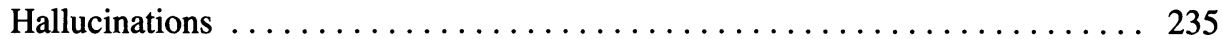

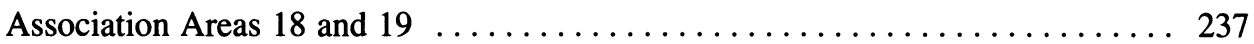

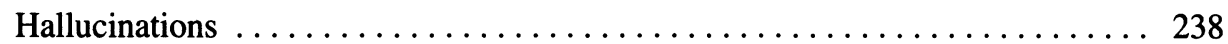

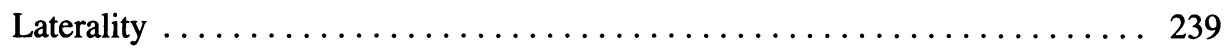

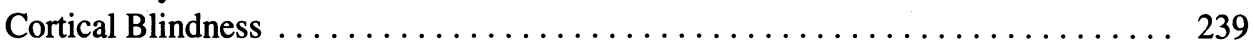

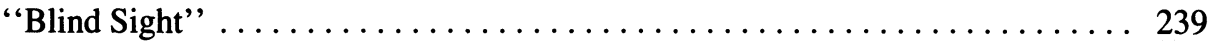

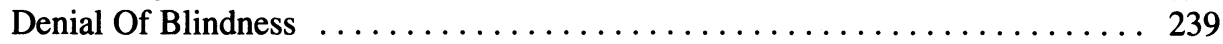

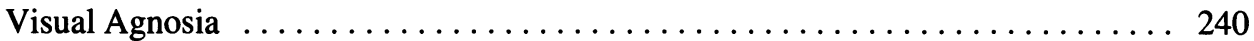

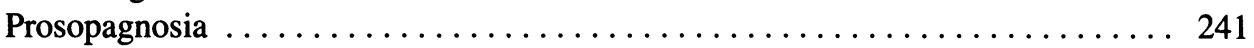

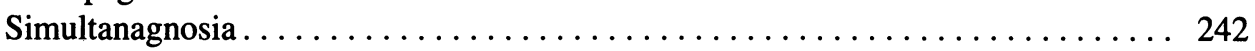

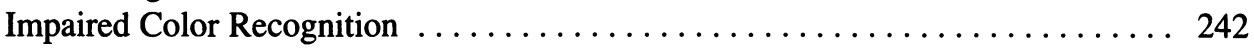

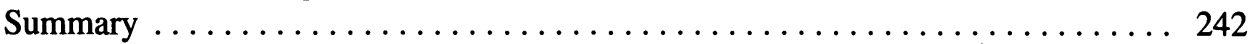

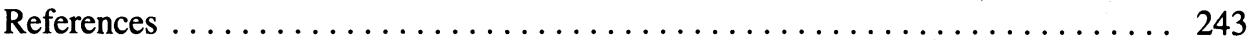

\section{Chapter 7}

The Temporal Lobes $\ldots \ldots \ldots \ldots \ldots \ldots \ldots \ldots \ldots \ldots \ldots \ldots \ldots \ldots$

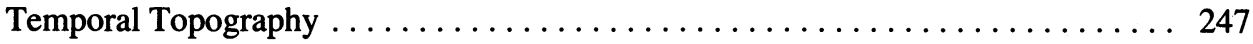

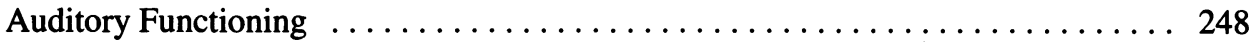

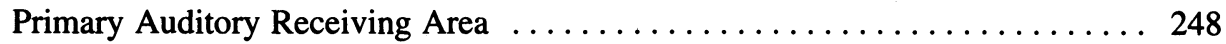

Temporal-Sequential and Linguistic Sensitivity ................ 250

Spatial Localization, Attention, and Environmental Sounds ........... 251

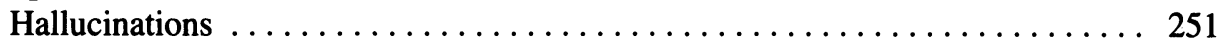

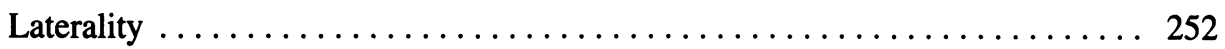

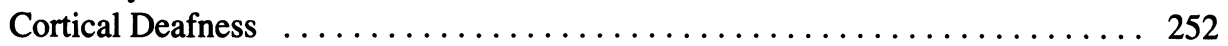

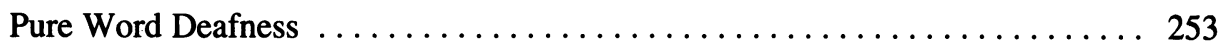

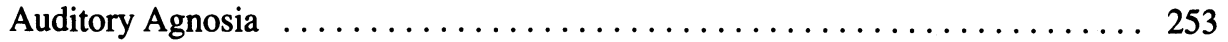

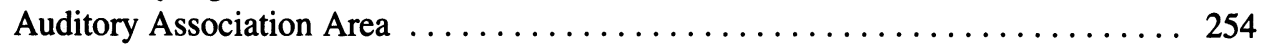

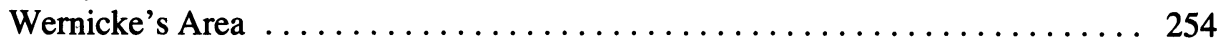

Receptive Aphasia ............................. 255

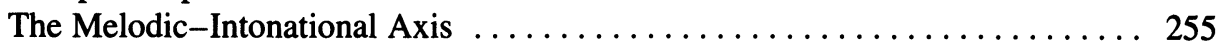




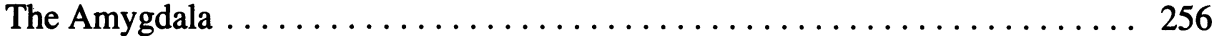

Middle Temporal Lobe $\ldots \ldots \ldots \ldots \ldots \ldots \ldots \ldots \ldots \ldots \ldots \ldots \ldots \ldots . \ldots \ldots$

Anterior Middle Temporal Lobe: Audition . . . . . . . . . . . . . . . . . 257

Left ATM Language Capabilities . . . . . . . . . . . . . . . . . . 257

Hallucinations ..................................... 257

Posterior Middle Temporal Lobe: Vision $\ldots \ldots \ldots \ldots \ldots \ldots \ldots \ldots \ldots \ldots$

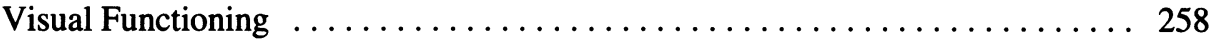

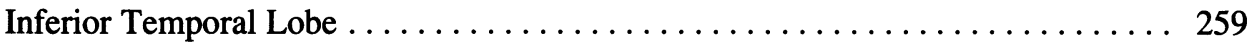

Visual Capabilities and Form Recognition $\ldots \ldots \ldots \ldots \ldots \ldots \ldots \ldots \ldots$

Visual Attention .................................. 260

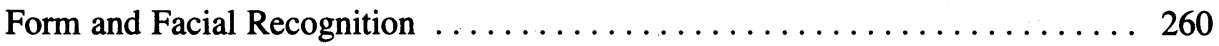

Prosopagnosia and Visual Discrimination Deficits ............... 261

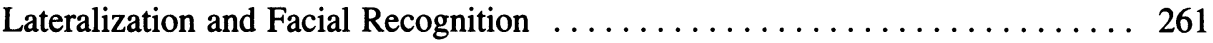

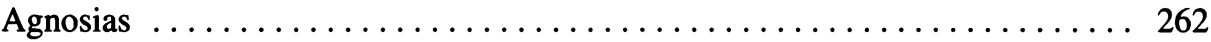

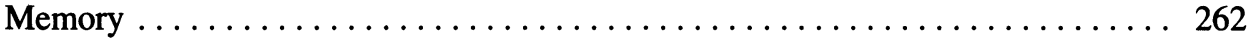

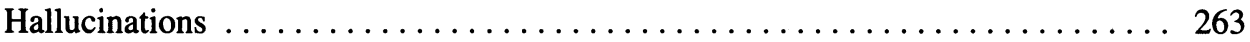

Hallucinations and the Interpretation of Neural "Noise" . . . . . . . . . 263

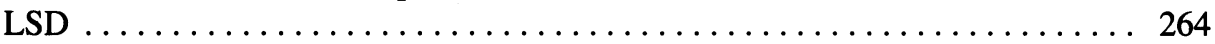

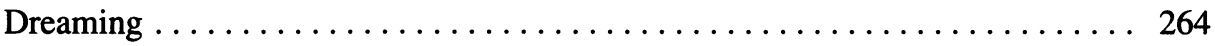

Temporal Lobe (Partial Complex) Seizures . . . . . . . . . . . . . . . 265

Personality and Psychiatric Disturbances ................... 265

Behavioral Manifestations of Seizures . . . . . . . . . . . . . . . . . . . . 266

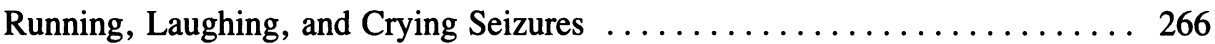

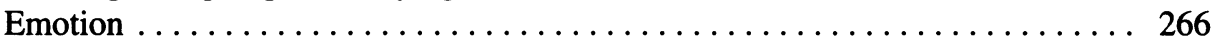

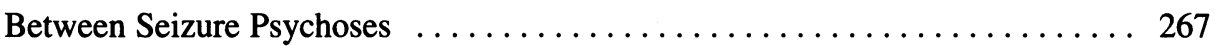

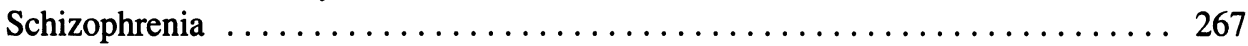

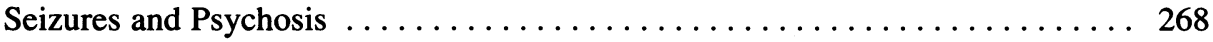

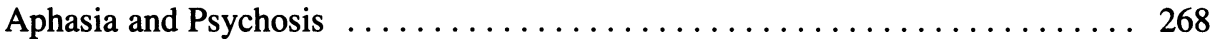

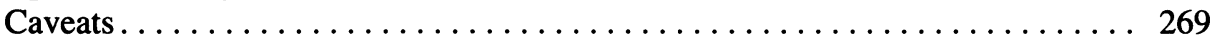

References .................................. 269

\section{Chapter 8}

Cerebral and Cranial Trauma: Anatomy and Pathophysiology of Mild,

Moderate, and Severe Head Injury ................ 277

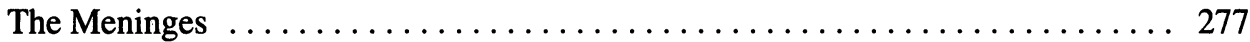

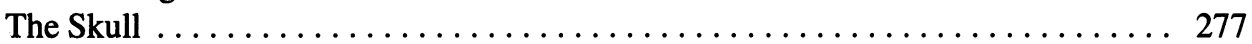

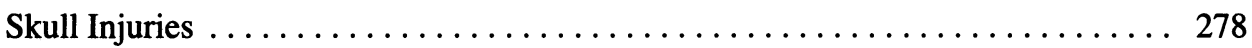

Basilar Fractures .................................... 279

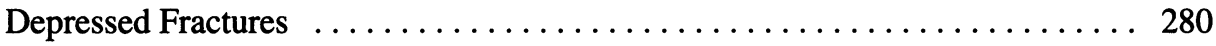

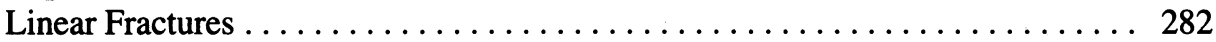

Anosmia ...................................... 284

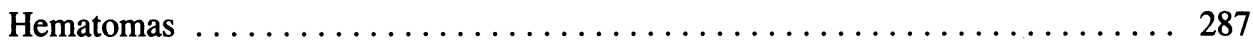

Intradural Hematomas . . . . . . . . . . . . . . . . . . . . . . . . . . . 287

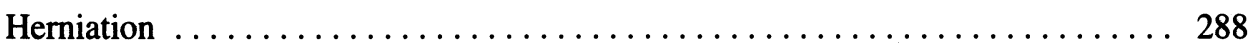

Falx, Cerebellar, and Temporal Lobe Herniations $\ldots \ldots \ldots \ldots \ldots \ldots . \ldots 288$ 


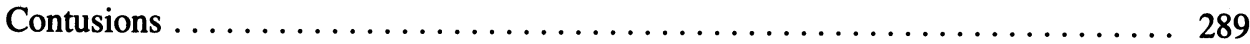

Necrosis ....................................... 290

Consciousness ................................. 290

Coup and Contrecoup Contusions $\ldots \ldots \ldots \ldots \ldots \ldots \ldots \ldots \ldots \ldots \ldots \ldots$

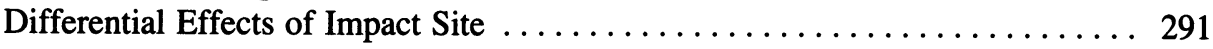

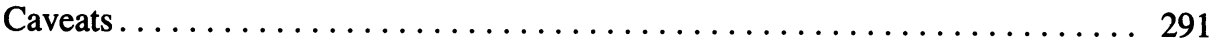

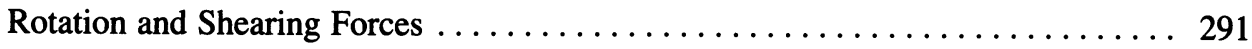

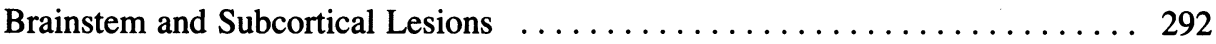

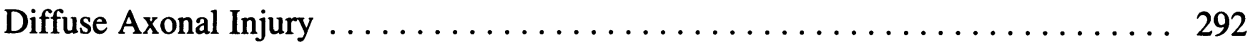

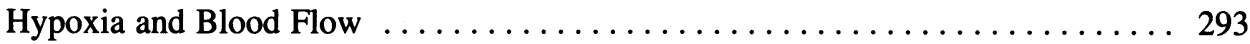

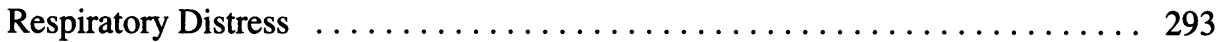

Vascular Trauma ................................... 294

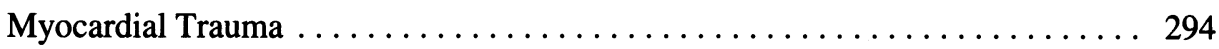

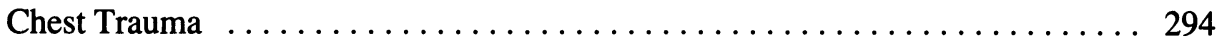

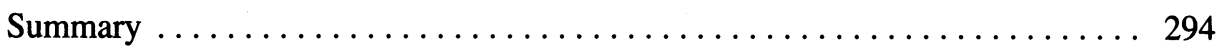

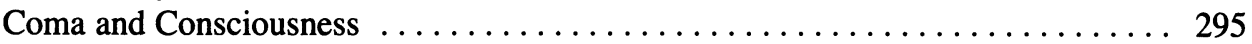

Reticular Damage and Immediate Cessation of Consciousness ......... 295

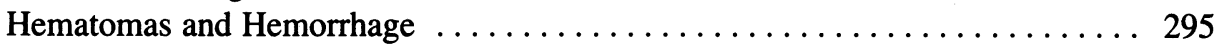

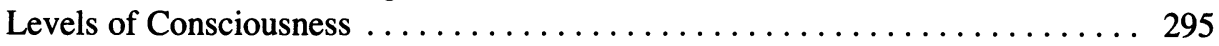

The Glasgow Coma Scale ............................ 296

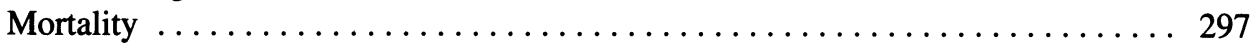

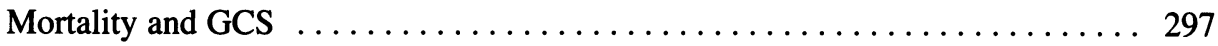

Mortality and Age ............................... 298

Mortality and Motor Functioning $\ldots \ldots \ldots \ldots \ldots \ldots \ldots \ldots \ldots \ldots \ldots \ldots$

General Complications . . . . . . . . . . . . . . . . . . . . . . . . . . . . . . 298

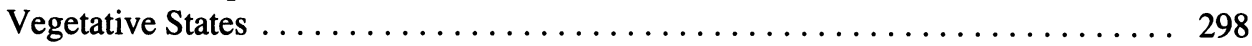

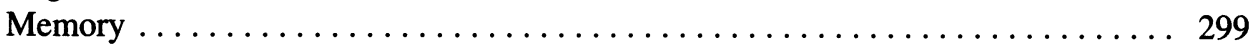

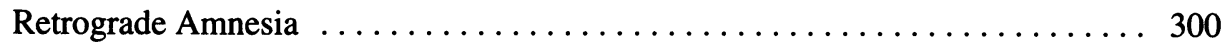

Post-Traumatic/Anterograde Amnesia . . . . . . . . . . . . . . . 300

Caveats..................................... 301

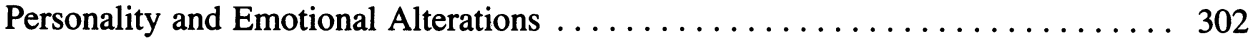

Delayed Development of Emotional Changes . . . . . . . . . . . . . . . 302

Family Stress . . . . . . . . . . . . . . . . . . . . . . . . . . . . . . . . . . . . 302

Premorbid Personality ........................... 303

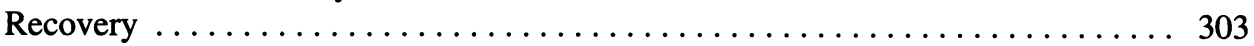

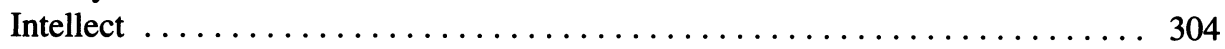

Employment.................................. 304

Age......................................... 304

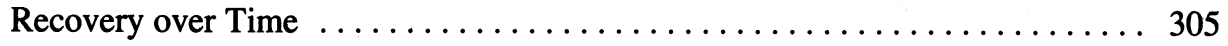

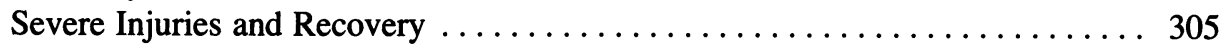

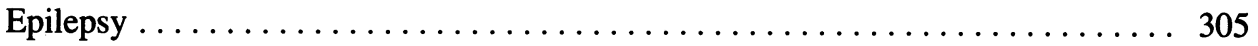

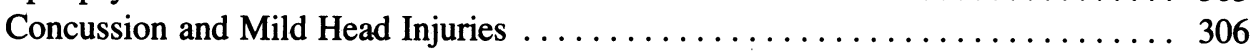

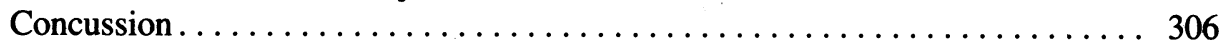

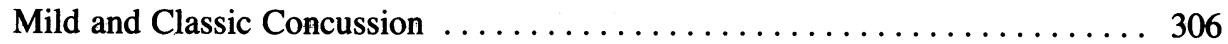

Mild Head Injury . . . . . . . . . . . . . . . . . . . . . . . . 307

Brainstem Abnormalities . . . . . . . . . . . . . . . . . . . . . . . . . . 308

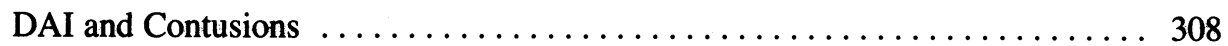


Neuropsychological Deficits $\ldots \ldots \ldots \ldots \ldots \ldots \ldots \ldots \ldots \ldots \ldots \ldots \ldots \ldots . \ldots . \ldots . \ldots$

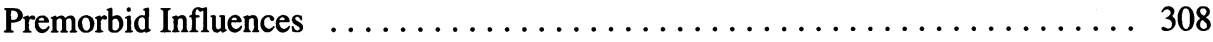

Postconcussion Syndrome . . . . . . . . . . . . . . . . . . . . 309

Emotional Sequelae . . . . . . . . . . . . . . . . . . . . . . . 309

Cerebral Blood Flow . . . . . . . . . . . . . . . . . . . . . . . . 310

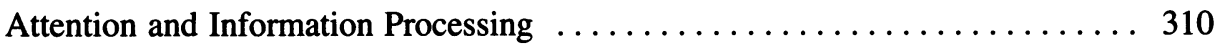

Whiplash and Blood Flow ........................ 310

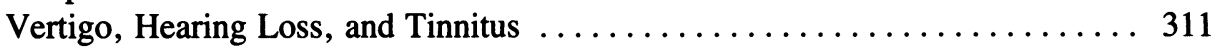

Diploplia and Photophobia ......................... 311

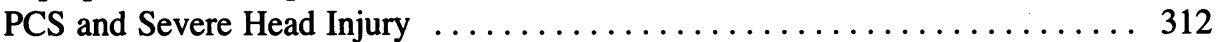

Premorbid Characteristics . . . . . . . . . . . . . . . . . . . 312

References ..................................... 313

\section{Chapter 9}

Stroke and Cerebrovascular Disease $\ldots \ldots \ldots \ldots \ldots \ldots \ldots \ldots \ldots$

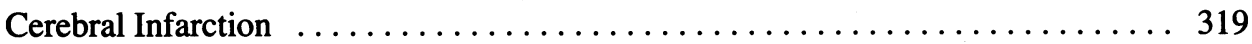

Functional Anatomy of the Heart and Arterial Distribution ........... 320

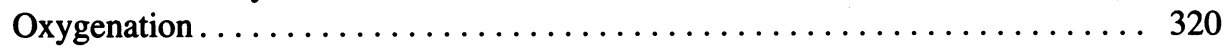

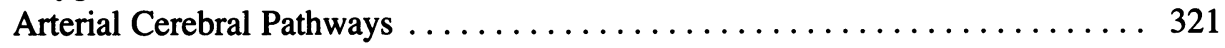

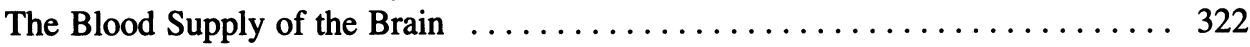

The Carotid System . . . . . . . . . . . . . . . . . . . . 322

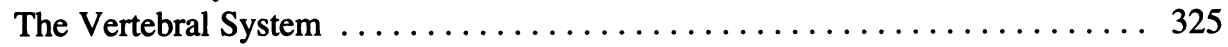

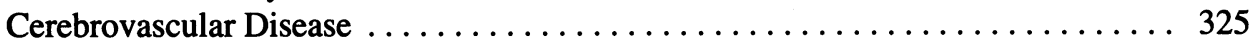

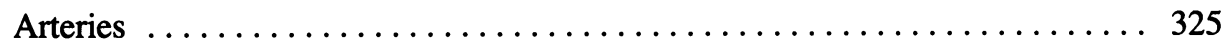

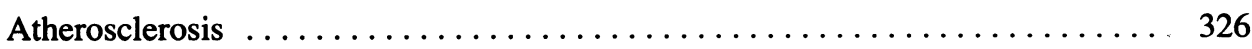

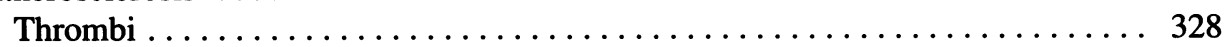

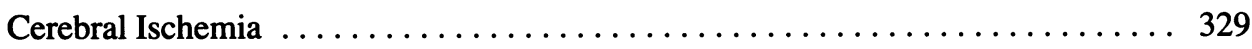

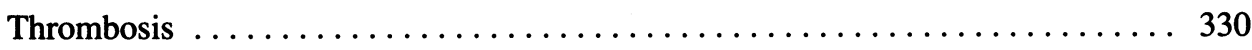

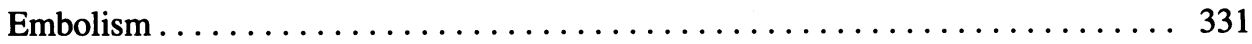

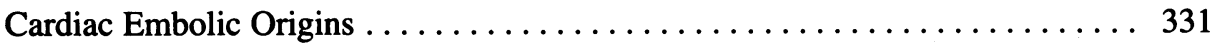

The Carotid and Left Middle Cerebral Artery ................. 331

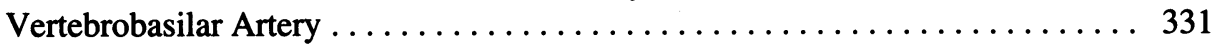

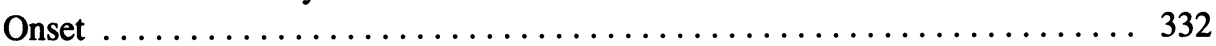

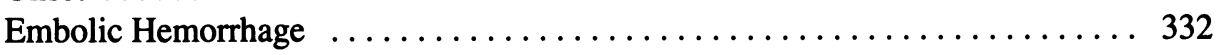

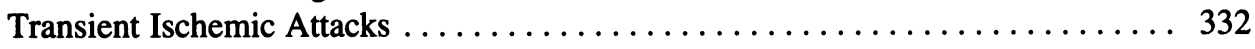

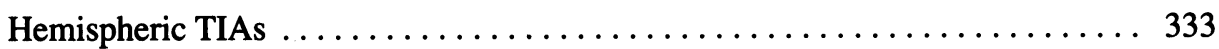

Vertebrobasilar TIAs ............................. 333

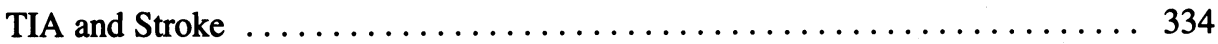

Lacunar Strokes . . . . . . . . . . . . . . . . . . . . . . . . . 334

Multi-Infarct Dementia . . . . . . . . . . . . . . . . . . . . . 334

Heart Disease, Myocardial Infarction, and Cardiac Surgery $\ldots \ldots \ldots \ldots \ldots . \ldots 35$

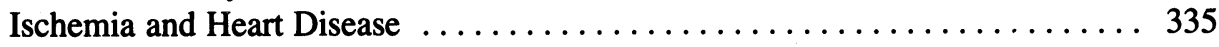

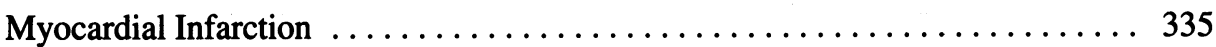

Global Ischemia . . . . . . . . . . . . . . . . . . . 336 


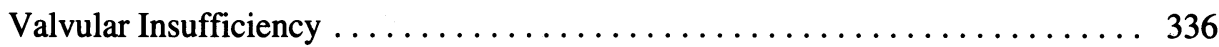

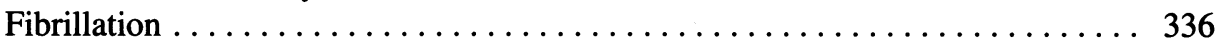

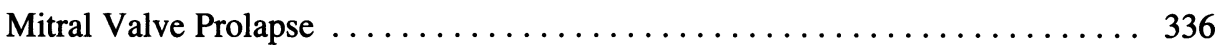

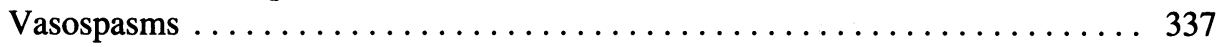

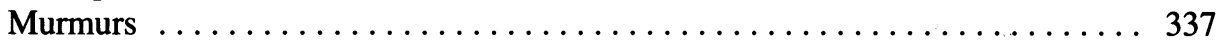

Cardiac Surgery . . . . . . . . . . . . . . . . . . . . . . . . . . . . . 337

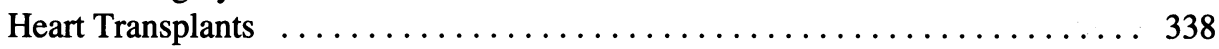

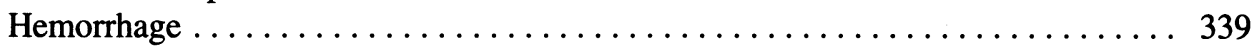

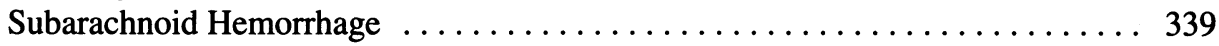

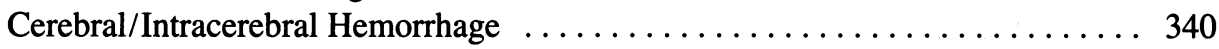

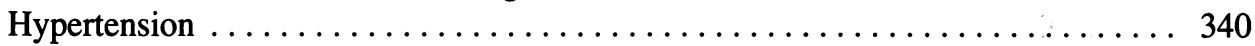

Aneurysms, AVMs, Tumors, Amyloid Angiopathy $\ldots \ldots \ldots \ldots \ldots \ldots \ldots \ldots . \ldots \ldots 1$

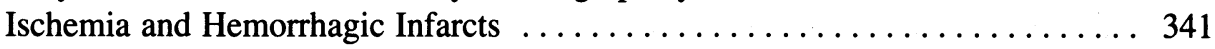

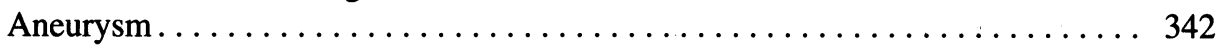

Arteriovenous Malformations $\ldots \ldots \ldots \ldots \ldots \ldots \ldots \ldots \ldots \ldots \ldots \ldots \ldots \ldots \ldots$

Drug-Induced Hemorrhages $\ldots \ldots \ldots \ldots \ldots \ldots \ldots \ldots \ldots \ldots \ldots . \ldots \ldots \ldots$

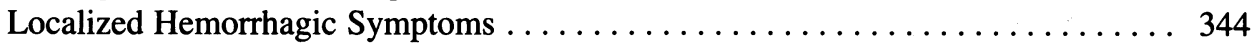

Hemorrhages and Strokes: Arterial Syndromes $\ldots \ldots \ldots \ldots \ldots \ldots \ldots \ldots \ldots . \ldots \ldots 45$

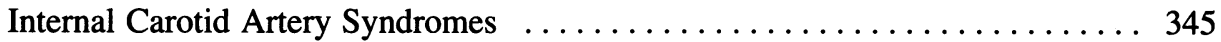

Middle Cerebral Artery Syndromes . . . . . . . . . . . . . . . . 345

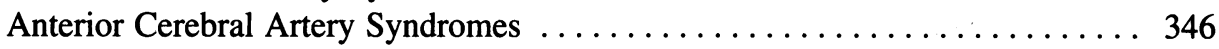

Vertebrobasilar Artery Syndromes $\ldots \ldots \ldots \ldots \ldots \ldots \ldots \ldots \ldots \ldots \ldots \ldots . \ldots \ldots$

Lateral Medullary Syndrome $\ldots \ldots \ldots \ldots \ldots \ldots \ldots \ldots \ldots \ldots \ldots \ldots \ldots \ldots \ldots$

Medial Medullary Syndrome $\ldots \ldots \ldots \ldots \ldots \ldots \ldots \ldots \ldots \ldots \ldots \ldots \ldots . \ldots \ldots$

Basilar Artery Syndromes . . . . . . . . . . . . . . . . . . . . . . . . 349

Locked-in Syndrome . . . . . . . . . . . . . . . . . . . . . . . . . . . . . . . 349

Posterior Cerebral Artery Syndromes . . . . . . . . . . . . . . . . . . . . 349

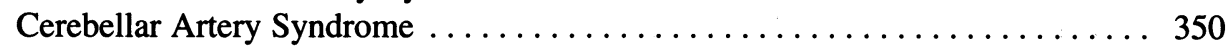

Differential Diagnosis: Carotid versus Vertebral System ............ 350

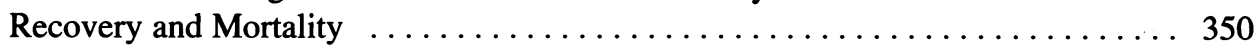

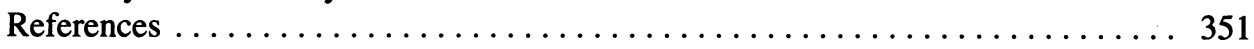

Chapter 10

Cerebral Neoplasms ................................... 355

Tumor Development: Genetics $\ldots \ldots \ldots \ldots \ldots \ldots \ldots \ldots \ldots \ldots \ldots \ldots \ldots \ldots$

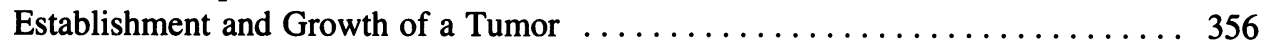

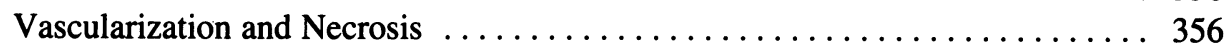

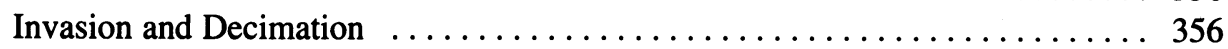

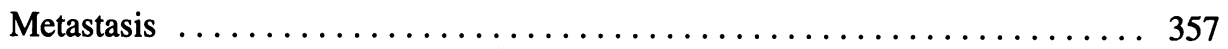

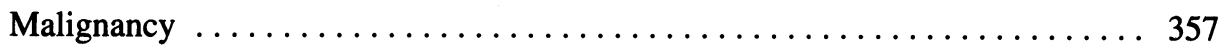

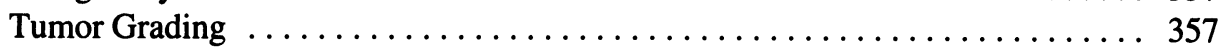

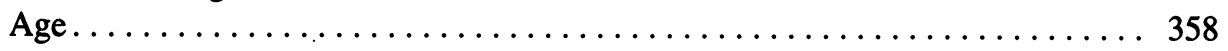

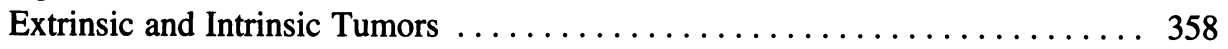


Neoplasms and Symptoms Associated with Tumor Formation $\ldots \ldots \ldots \ldots \ldots 35$

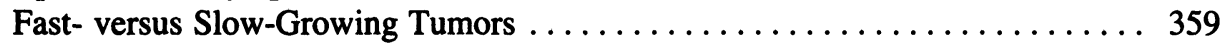

Generalized Neoplastic Symptoms . . . . . . . . . . . . . . . . 359

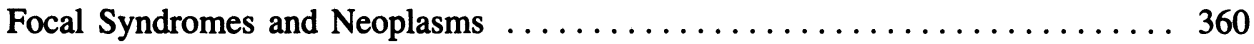

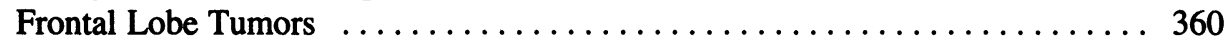

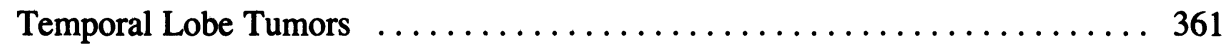

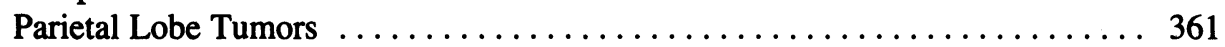

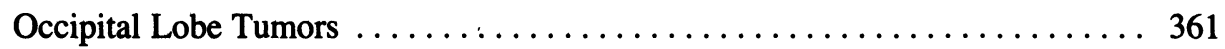

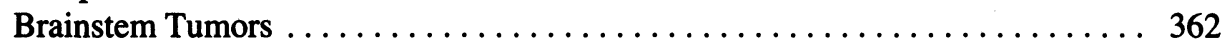

Cerebellar Tumors .............................. 362

Tumors of the Ventricles: Ependymomas $\ldots \ldots \ldots \ldots \ldots \ldots \ldots \ldots \ldots \ldots \ldots \ldots \ldots$

Medulloblastoma ............................... 362

Tumors of the Pineal Gland: Pinealmomas .................... 363

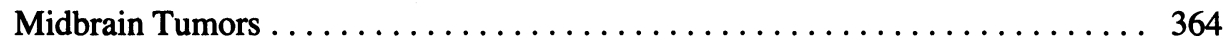

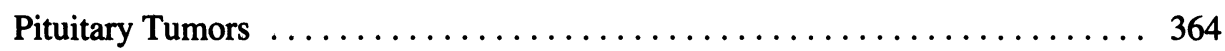

Tumors of the Meninges: Meningioma $\ldots \ldots \ldots \ldots \ldots \ldots \ldots \ldots \ldots \ldots \ldots$

Acoustic Neuromas and Schwannomas ................... 365

Astrocytoma and Glioblastoma Multiforme $\ldots \ldots \ldots \ldots \ldots \ldots \ldots \ldots \ldots 6$

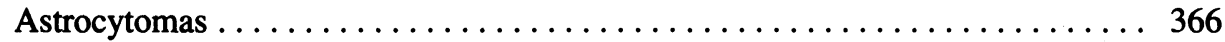

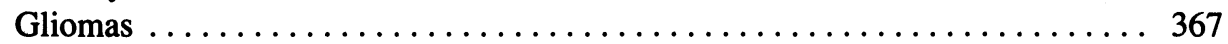

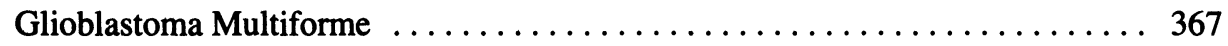

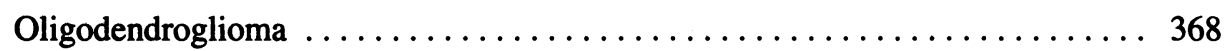

Lymphomas, Sarcomas, Neuroblastomas, Cysts . . . . . . . . . . . . 369

Lymphomas . . . . . . . . . . . . . . . 369

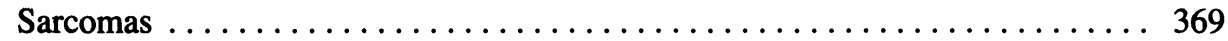

Neuroblastomas . . . . . . . . . . . . . . . . . . . . . . . . . . . . . . . . . 369

Cysts ........................................ 369

Unilateral Tumors and Bilateral Dysfunction $\ldots \ldots \ldots \ldots \ldots \ldots \ldots \ldots \ldots$

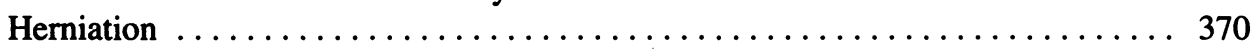

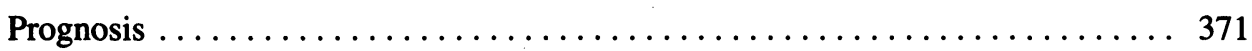

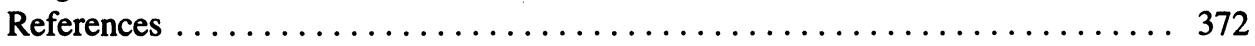

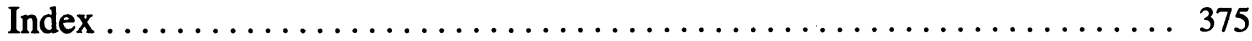




\section{Brain Plates}




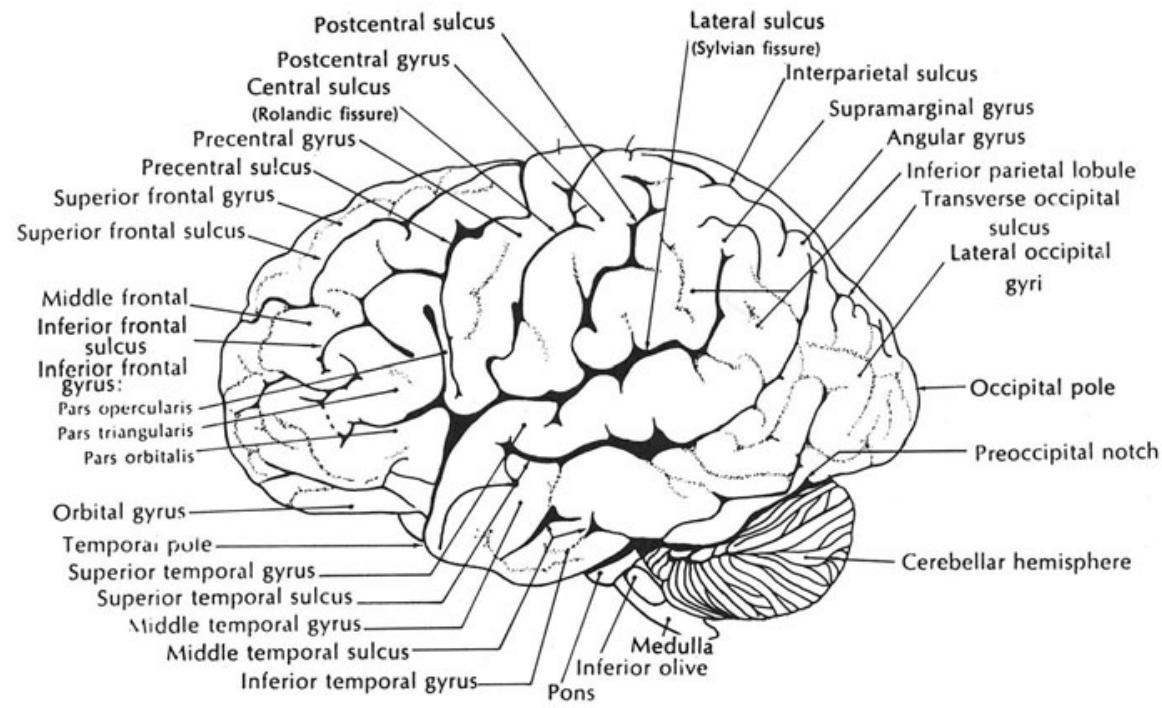

FIGURE 1. Lateral surface of the left cerebral hemisphere. From Structure of the human brain by S. J. DeArmond, M. M. Fusco, \& M. M. Dewey, 1976. New York: Oxford University Press. Reprinted by permission. 


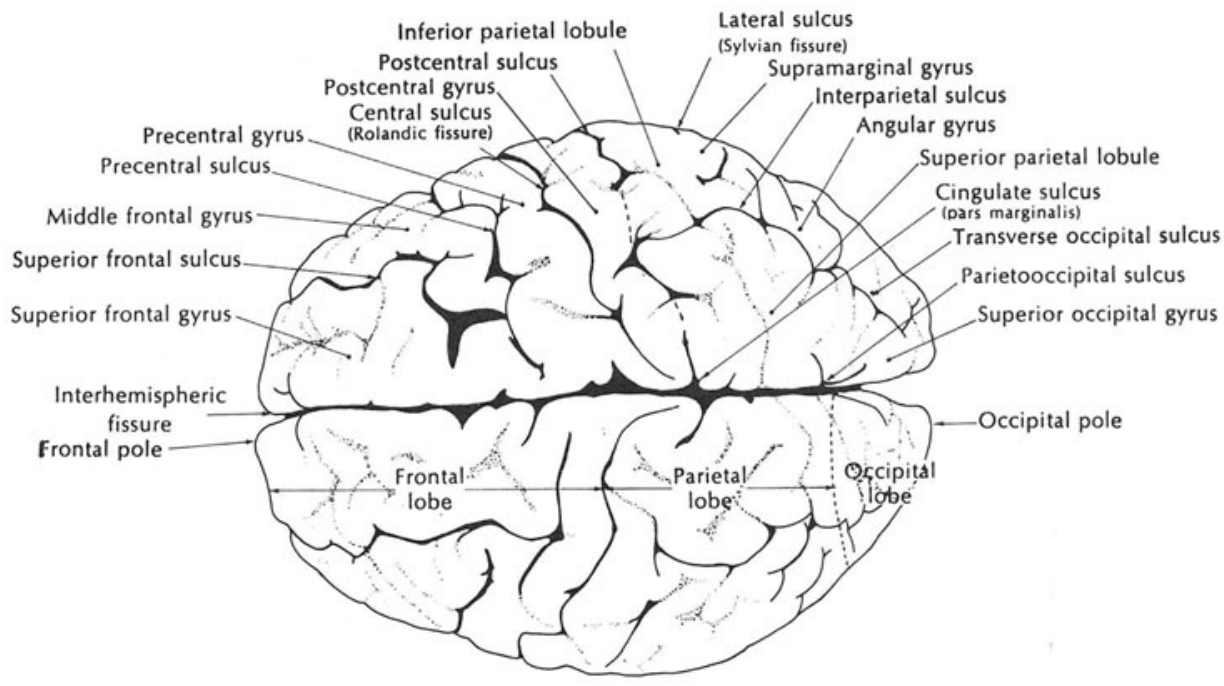

FIGURE 2. Superior surface of the brain. From Structure of the human brain by S. J. DeArmond, M. M. Fusco, \& M. M. Dewey, 1976. New York: Oxford University Press. Reprinted by permission. 


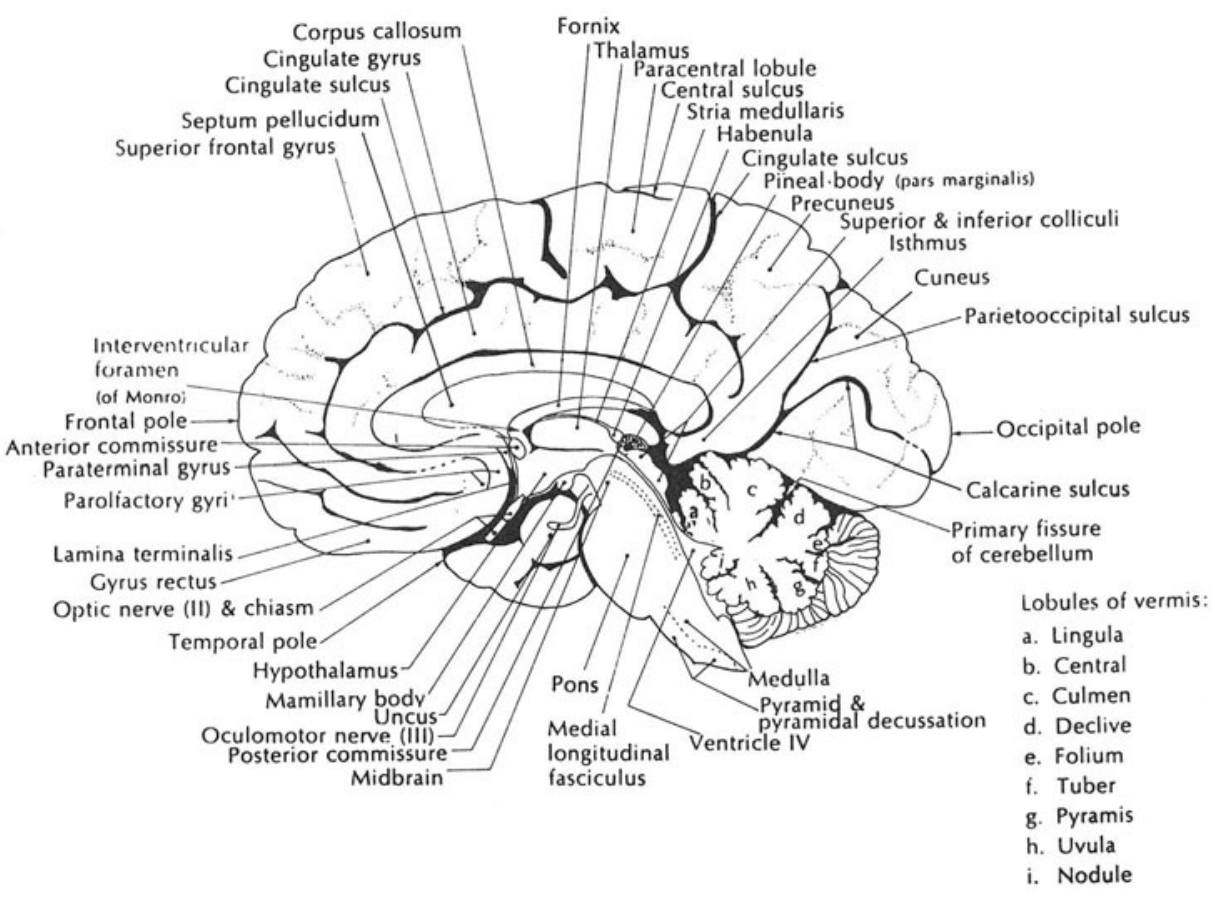

FIGURE 3. Medial (split-brain) view of the right half of the brain. From Structure of the human brain by S. J. DeArmond, M. M. Fusco, \& M. M. Dewey, 1976. New York: Oxford University Press. Reprinted by permission. 


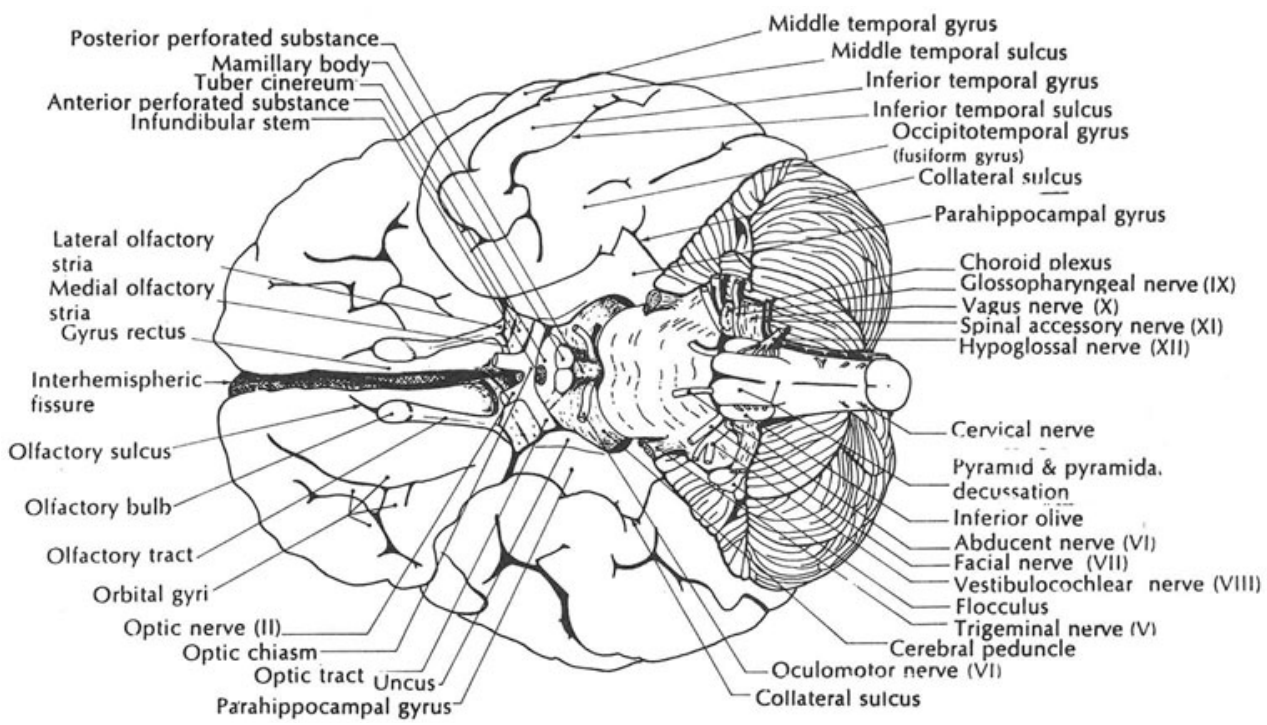

FIGURE 4. Inferior surface of the brain. From Structure of the human brain by S. J. DeArmond, M. M. Fusco, \& M. M. Dewey, 1976. New York: Oxford University Press. Reprinted by permission. 


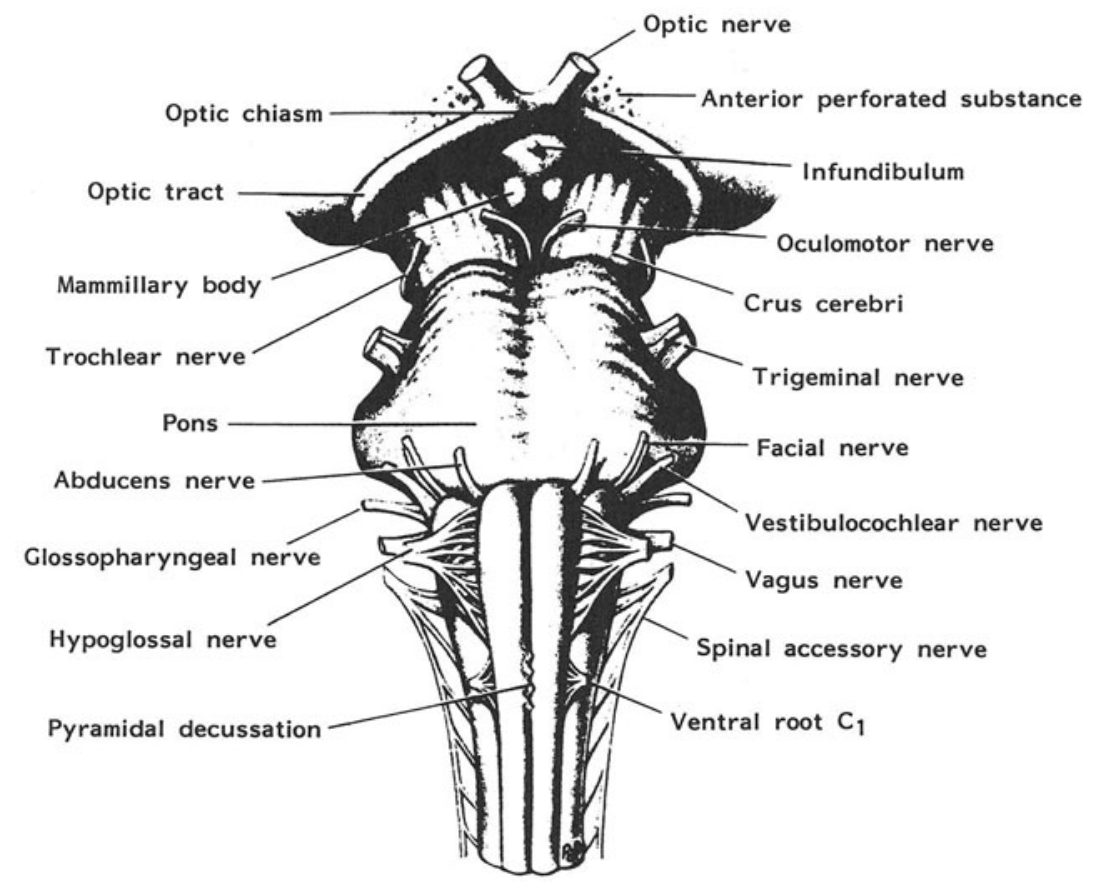

FIGURE 5. The anterior aspect of the brainstem and midbrain. From Human neuroanatomy by R. C. Truex \& M. B. Carpenter, 1969. Courtesy of The Williams \& Wilkins Co., Baltimore, MD. 


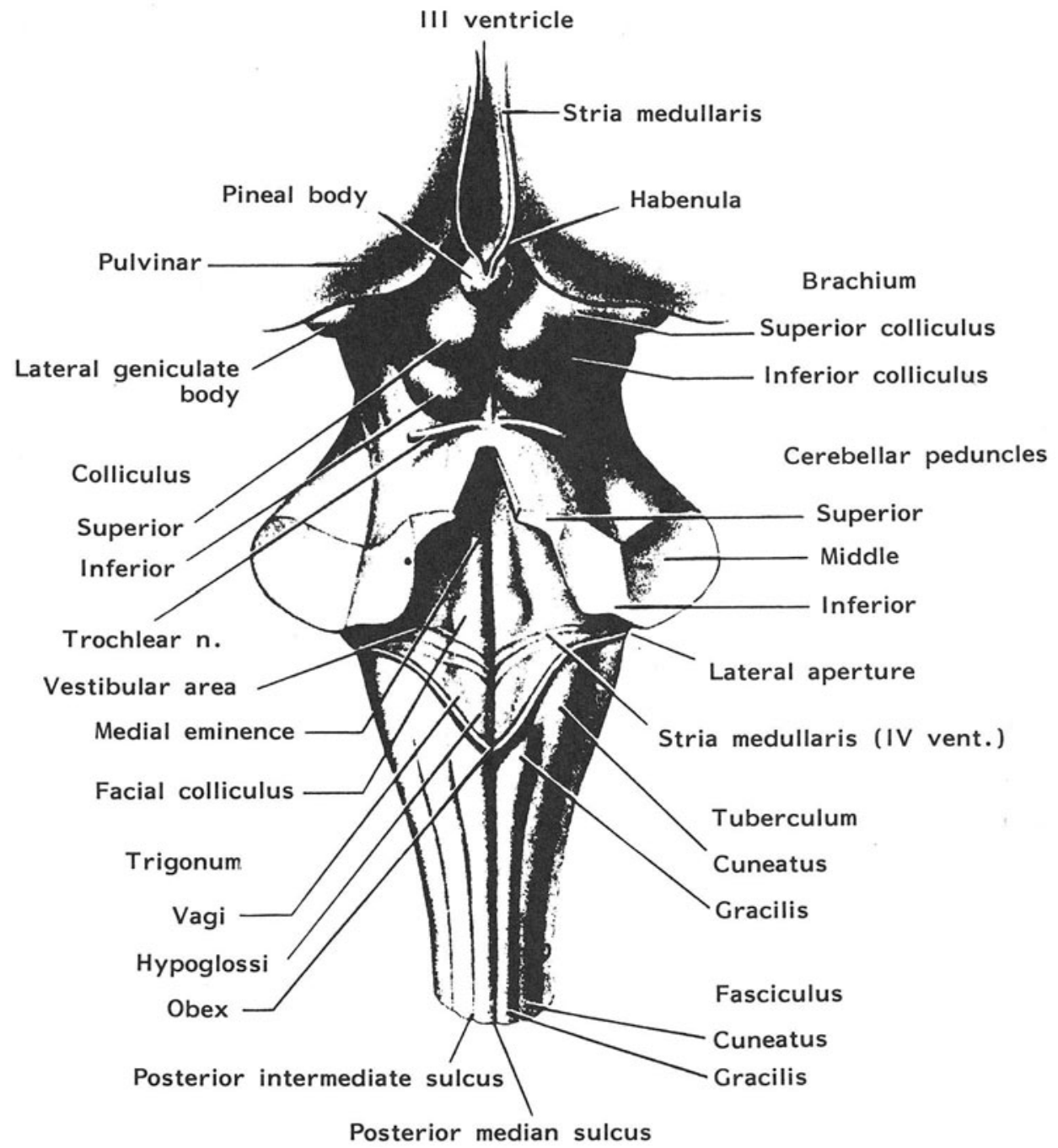

FIGURE 6. The posterior aspect of the brainstem with the cerebellum removed. From Human neuroanatomy by R. C. Truex \& M. B. Carpenter, 1969. Courtesy of The Williams \& Wilkins Co., Baltimore, MD. 


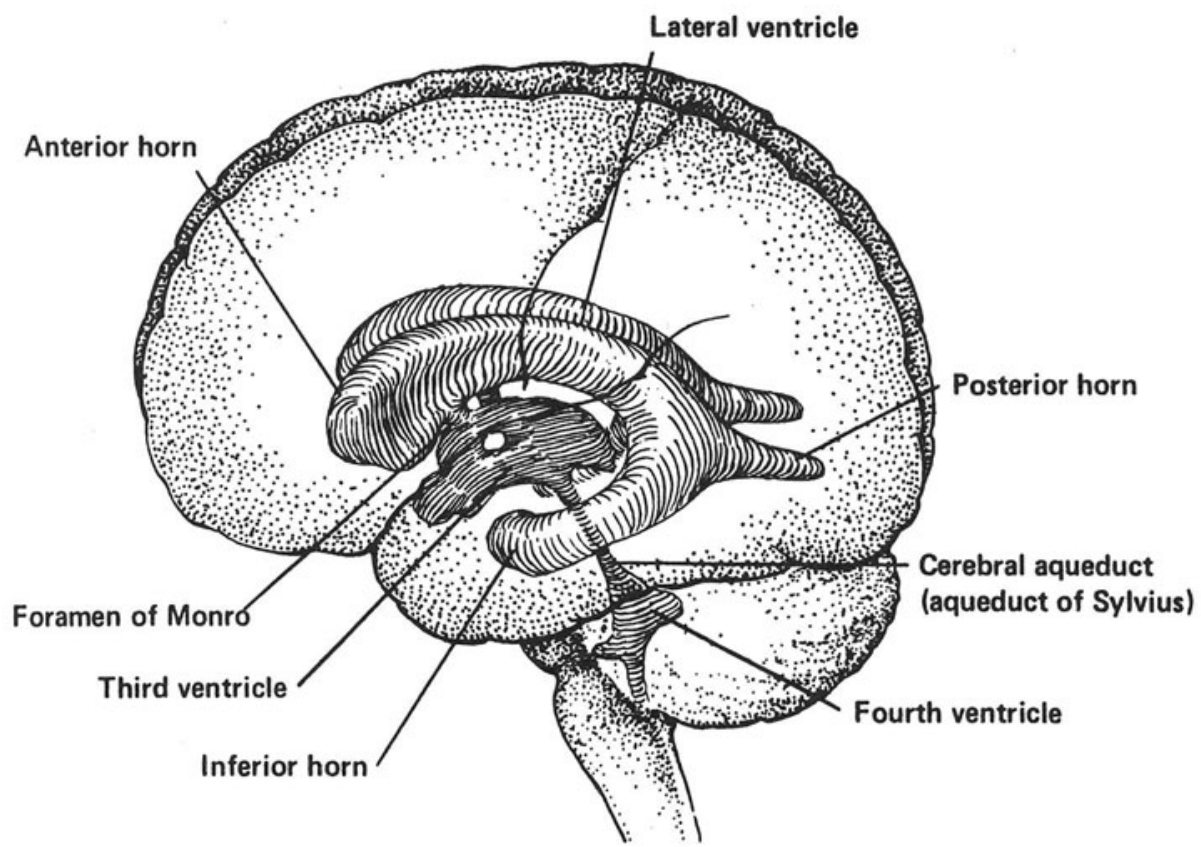

FIGURE 7. The ventricular system of the brain. From Correlative neuroanatomy, 20th Ed. by J. DeGroot \& J. G. Chusid, 1988. Courtesy of Appleton \& Lange, E. Norwalk, CT. 